\title{
Molecular imaging reveals biodistribution of P-cadherin LP- DART bispecific and trafficking of adoptively transferred $T$ cells in mouse xenograft model
}

\author{
Vijay R. Gupta ${ }^{1}$, Adam Root ${ }^{2}$, Timothy Fisher ${ }^{3}$, Rand Norberg ${ }^{1}$, John David ${ }^{1}$, Tracey \\ Clark $^{4}$, Justin Cohen ${ }^{2}$, Chad May ${ }^{3}$ and Anand Giddabasappa ${ }^{1}$ \\ ${ }^{1}$ Global Science \& Technology (GST) - Comparative Medicine, Pfizer Global Research Development and Medical, San Diego, \\ CA 92121, USA \\ ${ }^{2}$ BioMedicine Design, Cambridge, MA 02139, USA \\ ${ }^{3}$ Oncology Research and Development, San Diego, CA 92121, USA \\ ${ }^{4}$ PDM Biotherapeutics, Pfizer Inc., San Diego, CA 92121, USA \\ Correspondence to: Anand Giddabasappa, email: anand.giddabasappa@pfizer.com \\ Keywords: bispecific antibody; tumor targeting; biodistribution; T cells; molecular imaging \\ Received: November 03, $2019 \quad$ Accepted: March 14, $2020 \quad$ Published: April 14, 2020
}

Copyright: Gupta et al. This is an open-access article distributed under the terms of the Creative Commons Attribution License 3.0 (CC BY 3.0), which permits unrestricted use, distribution, and reproduction in any medium, provided the original author and source are credited.

\section{ABSTRACT}

P-cadherin-LP-DART is a bispecific antibody targeting P-cadherin expressed on the tumor cells and CD3 on the T-cells. Previously we demonstrated the development and efficacy of P-cadherin-LP-DART in in vitro and in vivo models. Here, we evaluated the three pillars: exposure, targeting specificity and pharmacodynamic modulation for P-cadherin-LP-DART using fluorescence molecular tomography (FMT). Bispecific antibodies and T-cells were conjugated with a near-infrared fluorophores: VivoTag ${ }^{\circledR} 680 X \mathrm{X}$ (VT680) and CellVue ${ }^{\circledR}$ NIR815 (CV815), respectively. In vitro binding and cytotoxic T-lymphocyte assay demonstrated that P-cadherin-LP-DART significantly retained its properties after VT680 conjugation. In vivo FMT imaging was performed to determine the bispecific biodistribution and T-cell trafficking in HCT116 xenograft model. Peak tumor exposure (2.71\%ID) was observed at 96 hr postinjection with measurable quantity even at $240 \mathrm{hr}$ (1.46\%ID) (Pillar 1). P-cadherinLP-DART accumulation in tumor was 20-25 fold higher compared to Control-LP-DART demonstrating the targeting specificity (Pillar 2). Imaging after engraftment of CV815 labeled T-cells showed P-cadherin-LP-DART mediated T-cell trafficking in tumors (Pillar 3). This study harnessed the multichannel capability of FMT and demonstrated the targeting of drug and trafficking of $T$ cells to tumors, simultaneously. Our results show the impact of molecular imaging in demonstrating three pillars of pharmacology, longitudinally and non-invasively.

\section{INTRODUCTION}

Antibody-based therapeutic platforms have revolutionized the biopharmaceutical landscape in last two decades [1]. Advances in protein engineering have transformed the antibody-based therapeutics to overcome limitations including antigen targeting, immunogenicity, pharmacokinetics and manufacturability $[1,2]$. Bispecific antibodies are one of these innovative platforms that can co-engage two different antigens at the same time $[2,3]$. Renewed interest in cancer immunotherapy has focused on bispecific antibodies as a preferred therapeutic modality used to direct immune effector cells to tumors expressing a target antigen to trigger an anti-tumor cytotoxic response $[2,4]$. Immune effector cells that can be engaged by bispecific antibodies include $\mathrm{T}$ cells, Natural Killer (NK) cells and Macrophages. Effector T cells have the ability to expand rapidly upon activation and also have the potential to generate immunological memory, thereby generating robust and durable anticancer response [5]. Mechanistically, bispecific antibodies targeting effector T cells co-engage the CD3 epsilon subunit on the T cells 
and specific antigen ( $\mathrm{s}$ ) on tumor cells to form a bispecific mediated synapse. Target co-engagement by bispecific antibodies leads to activation of the T cell receptor (TCR) signaling cascade resulting in release of granzymes and perforin molecules that results in tumor cytotoxicity. Currently, two $\mathrm{T}$ cell targeting bispecific antibodies have been approved to treat cancer and an additional 50 molecules are in different stages of clinical development $[3,6-8]$.

Bispecific antibodies with affinity to multiple targets offer greater functionality when compared to traditional monoclonal antibodies. However, development of these next generation biologics is increasingly complex and often requires intricate protein engineering approaches to create novel scaffolds and technology platforms. The bispecific antibody formats can be broadly categorized as a) conventional $\operatorname{IgG}$ like bispecific antibodies that retain Fc-mediated effector antibody-dependent cellular cytotoxicity (ADCC) and complement-dependent cytotoxicity (CDC) functionalities or b) novel non-IgG like bispecific antibodies that generally have dual binding functionality with minimal or no effector functions $[4,7]$. The dual-affinity re-targeting (DART) platform is a bispecific molecular format that can co-engage the TCR/ CD3 complex on effector T cells and a targeting antigen on tumor cells [9]. The DART format is a bispecific diabody comprised of two distinct antigen-specific single chain variable fragments $(\mathrm{scFv})$ joined on either two separate chains or as a single chain. The LP-DART format fuses this bispecific diabody to an engineered human IgG1 Fc domain that is devoid of Fc mediated effector functions (Figure 1A-1D) [10-12].

$\mathrm{P}$-cadherin is a classical cadherin that is part of the adherens junction and mediates calcium dependent cellcell adhesion. Differential upregulation of P-cadherin is observed in a certain solid cancer types including breast, gastric, endometrial, colorectal and pancreatic cancers when compared to normal tissues $[13,14]$. P-cadherin upregulation has also been shown to correlate with poor survival of breast cancer patients [15]. Thus P-cadherin is an attractive antigen to target tumors with elevated expression of this protein. P-cadherin LP-DART is a bispecfic molecule that uses the DART platform to bind CD3 epsilon on the T cells and P-cadherin on the tumor cells. The human Fc region of P-cadherin LPDART molecule is engineered to eliminate Fc gamma receptor binding but retain neonatal Fc receptor $(\mathrm{FcRn})$ binding and thus offer extended plasma half-life $[10,16]$. P-cadherin LP-DART has demonstrated potent cytotoxic T-cell mediated tumor cell killing in in vitro and in vivo models [16]. To further expand our understanding of the pharmacology of P-cadherin LP-DART (i.e., the exposure at the target site and the recruitment of T cells in tumors), we have utilized fluorescence molecular tomography (FMT) imaging $[17,18]$. Longitudinal in vivo biodistribution, tissue exposure and tumor targeting of a P-cadherin LP-DART was assessed using FMT after labeling with near-infra red (NIR) fluorophore VivoTag $^{\circledR} 680 \mathrm{XL}$ (here after referred as VT680). Additionally, we explored the possibility of adopting FMT imaging to provide mechanistic insights by visualizing the $\mathrm{T}$ cell redistribution and tumor trafficking dynamics upon treatment with P-cadherin LP-DART.

\section{RESULTS}

\section{Evaluation of binding and functional properties of VT680 conjugated P-cadherin LP-DART}

The bispecific antibodies were labeled with amine-reactive fluorophore VT680 using NHS (N-hydroxysuccimide) chemistry. In vitro $\mathrm{P}$-cadherin and CD3 binding property and functional cytotoxic activity of the VT680 bispecific antibody conjugates were evaluated prior to the in vivo studies. P-cadherin LP-DART binds specifically to human $\mathrm{P}$-cadherin and $\mathrm{CD} 3 \varepsilon$ proteins, whereas the negative control (Control LP-DART) binds only to the human $\mathrm{CD} 3 \varepsilon$ protein. Fluorophore labeled P-cadherin LP-DART and Control LP-DART were compared with respective unlabeled counterparts for binding to soluble human P-cadherin and soluble human CD3 epsilon/delta (hCD3 $\varepsilon / \delta$ ) (Figure 2A and 2B). Dose-dependent binding curves demonstrate that VT680 labeling of P-cadherin LP-DART minimally affect binding to P-cadherin when compared to unlabeled antibody. The half maximal effective concentration (EC50) for P-cadherin binding was $0.92 \mathrm{nM}, 0.93 \mathrm{nM}$ and $1.42 \mathrm{nM}$ for unlabeled P-cadherin LP-DART, and for P-cadherin LP-DART-VT680 with degree of labeling (DOL) of 0.5 and 2.0, respectively. However, the VT680 labeling reduced the binding of P-cadherin LP-DART to soluble hCD3 $\varepsilon / \delta$ with EC50 values of $4.34 \mathrm{nM}, 11.47 \mathrm{nM}$ and $>100 \mathrm{nM}$ for unlabeled antibody, DOL of 0.5 and 2.0, respectively. This data suggested that the CD3 binding domain was more sensitive to VT680 labeling than the P-cadherin binding domain.

In vitro functional activity of the P-cadherin LPDART and P-cadherin DART was evaluated using the cytotoxic T lymphocyte (CTL) assay. The EC50 values were $0.3 \mathrm{pM}, 1.4 \mathrm{pM}$ and $6.2 \mathrm{pM}$ for the unlabeled P-cadherin LP-DART, DOL 0.5 and 1.0, respectively (Figure 2C). P-cadherin LP-DART-VT680 with DOL 2.0 still retained cytotoxic activity, whereas the unlabeled control-LP-DART of control-LP-DART-VT680 at DOL 2.0 did not show any cytotoxicity (Supplementary Figure 1). Similarly, the P-cadherin DART as single chain diabody $(\mathrm{scDb})$ showed a reduction in cytotoxic activity after conjugation to VT680 at DOL of 1 (Supplementary Figure 2A). These results suggested that VT680 labeling had a DOL dependent effect on cytotoxicity, but still retained potent (low pM) cell killing potential at DOL of 1.0 . 


\section{In vivo biodistribution and tumor targeting kinetics of P-cadherin LP-DART in HCT-116 xenograft model}

We have previously shown the differential expression of P-cadherin in variety of human tumor cell lines [10]. The biodistribution and tumor targeting kinetics of P-cadherin LP-DART was evaluated in high P-cadherin expressing HCT116 xenograft model by longitudinal FMT imaging. Representative three-dimensional (3D) whole-body (torso) images showing the time course for both P-cadherin LPDART and Control LP-DART are represented in Figure 3A. A 3D region of interest (ROI) was placed in the torso area to represent signal from the whole-body. A minimum background signal $(<5 \%)$ was observed from the cranial region and thus was not included in quantitation. Quantitation of fluorescence by FMT in whole-body (Figure 3B) and liver (Figure 3C) showed initial spike in signal at $24 \mathrm{hr}$ time point followed by gradual decrease in signal due to sustained clearance of the probes. A 3D ROI was placed around the heart region to quantify the heart. Since we did not anticipate any binding to the heart tissue we considered this fluorescence signal as a measurement for blood. Overall, the whole-body, heart and liver profiles were similar in both P-cadherin LP-DART and Control LP-DART groups (Figure 3B-3D). However, we observed a selective tumor accumulation of P-cadherin LP-DART that gradually

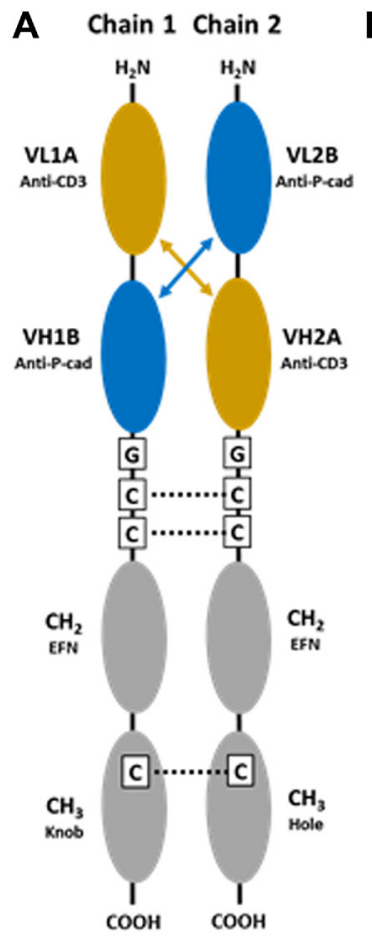

increased with time and peaked at $96 \mathrm{hr}$ post-injection (2.71\% Injected Dose (ID)) when compared to Control LP-DART which showed significantly lower accumulation $(0.67 \% \mathrm{ID})$ (Figure 3E). Although the tumor accumulation in both the groups declined after $96 \mathrm{hr}$, there was significant VT680 signal in the P-cadherin LP-DART group at $240 \mathrm{hr}$ post-injection (1.46\%ID) compared to Control LP-DART $(0.16 \% \mathrm{ID})$, suggesting a prolonged retention of the drug in the tumor. Overall, in vivo FMT imaging data showed selective tumor accumulation of P-cadherin LP-DART when compared to the non-specific Control LP-DART.

To compare the tumor targeting and clearance profiles of P-cadherin LP-DART and its $\mathrm{scDb}$ without $\mathrm{Fc}$ component, we evaluated whole-body biodistribution of P-cadherin DART-VT680 in HCT116 xenograft model (Supplementary Figure 2B and 2C). To our surprise, the whole-body profile of P-cadherin DART was different than the P-cadherin LP-DART. Significant accumulation of P-cadherin DART was observed in the kidneys as early as $6 \mathrm{hr}$ post-injection (Supplementary Figure 2C). The kidney signal was prominent compared to liver or the tumor suggesting that the P-cadherin DART is being cleared rapidly by the kidneys. Though moderate tumor accumulation $(\sim 1.5 \% \mathrm{ID}$, in vivo $)$ was observed at $48 \mathrm{hr}$ post-injection, as expected P-cadherin DART cleared rapidly by $96 \mathrm{hr}$ from the tumor $(<0.5 \% \mathrm{ID}$, in vivo) and the whole body (Supplementary Figure 2B and 2C).
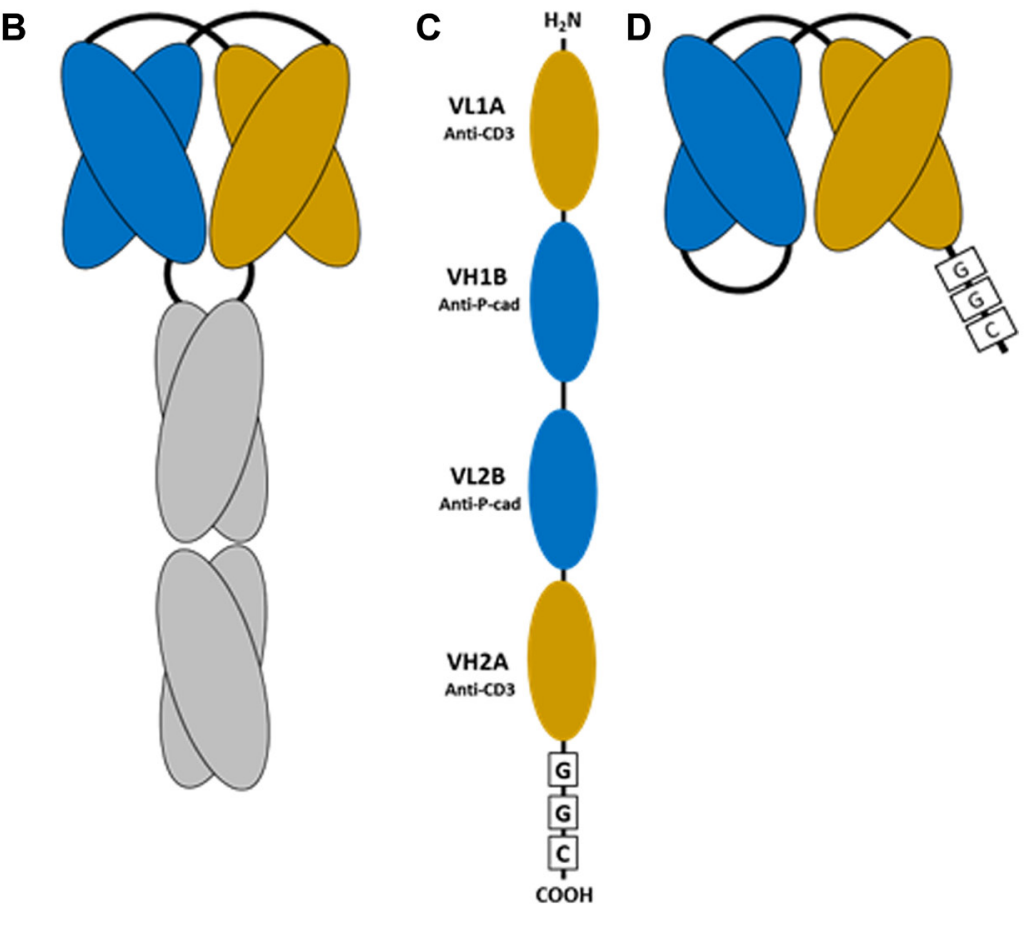

Figure 1: Schematic of the bispecific reagents used in this study. (A) Representation of the individual chains of anti-Pcadherin human IgG1 Fc-containing DART, also referred to as P-cadherin LP-DART. Cysteine residues introduced for interchain disulfide formation are indicated by $\mathrm{C}$. EFN, effector function null mutations in $\mathrm{CH} 2$. Knob, hole: complementary mutations introduced to force Fc heterodimerization [10-12]. (B) Schematic representation of the folded P-cadherin LP-DART protein after heterodimerization. (C) Representation of the anti-P-cadherin single chain diabody $(\mathrm{scDb})$ DART linear expression construct. Cysteine residue introduced for site specific conjugation at the C-terminus is indicated by C. (D) Schematic representation of the folded anti-P-cadherin DART (scDb) protein. 


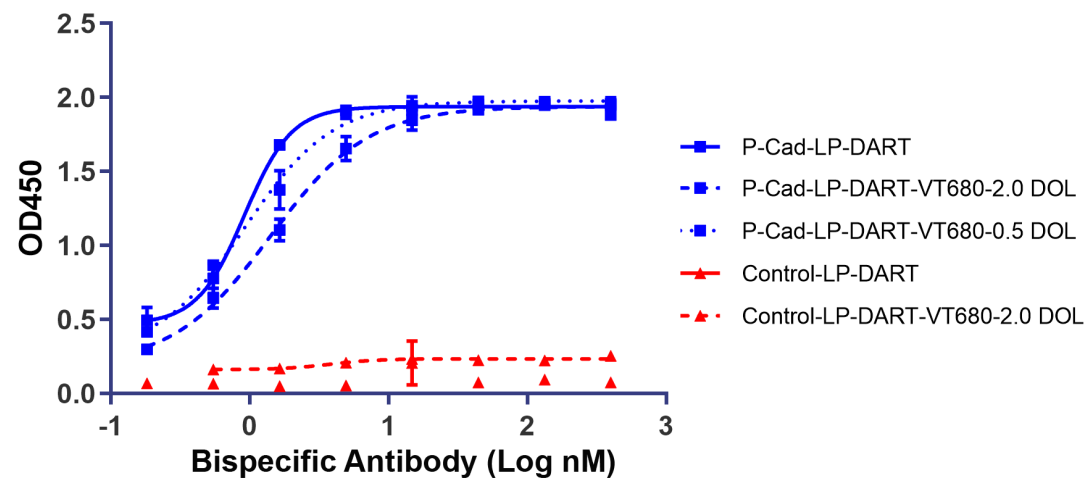

\section{B Soluble huCD3 $\varepsilon / \delta$ Binding ELISA}

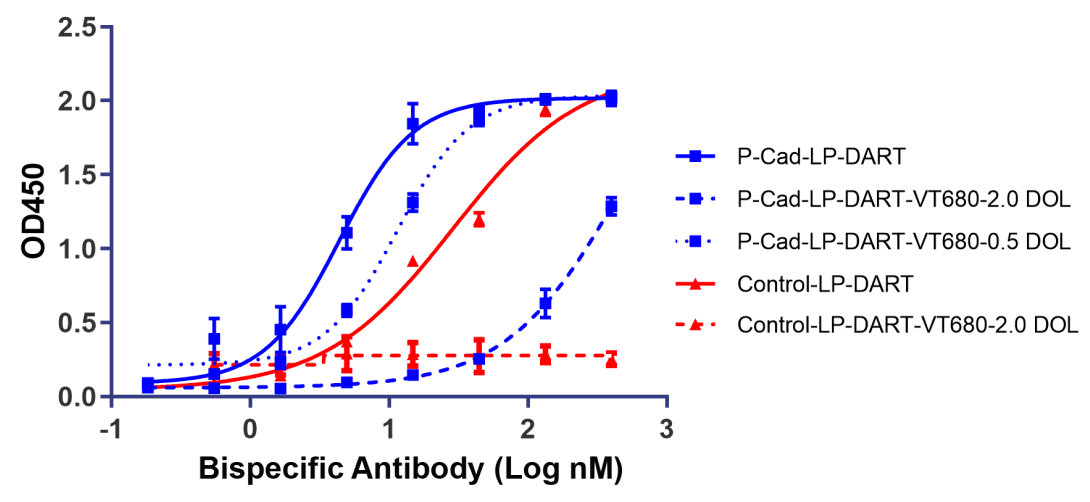

C Cytotoxicity Assay (5:1 E:T, 24 h)

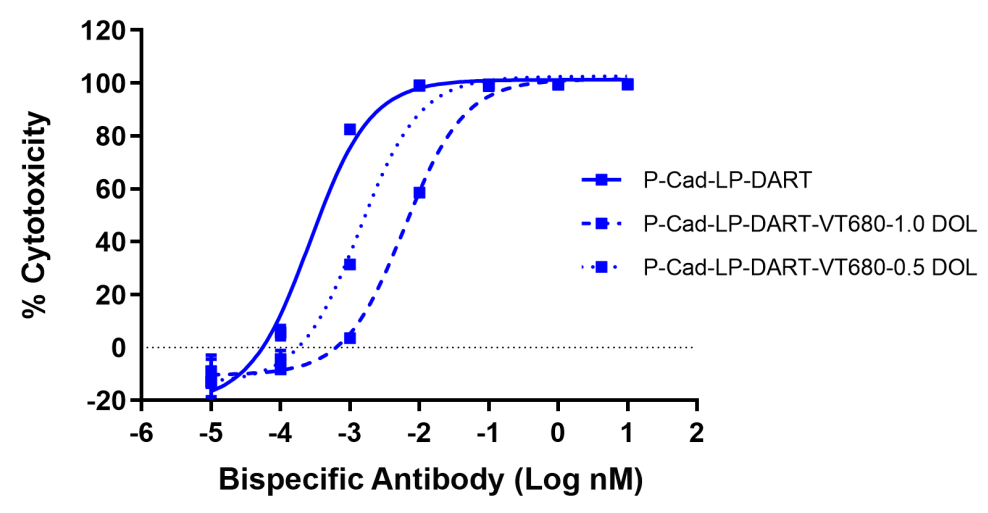

Figure 2: In vitro assays to evaluate the effect of labeling of VivoTag ${ }^{\circledR}$ 680XL to P-cadherin LP-DART. ELISA based assay was used to evaluate the effect of VT680 labeling on binding of P-cadherin LP-DART to human P-cadherin and human CD3. The proteins were coated on to the plates and incubated with serial dilutions of P-cadherin LP-DART-VT680 or Control LP-DART-VT680 for $1 \mathrm{hr}$ at $37^{\circ} \mathrm{C}$. The bound P-cadherin LP-DART was quantified using IgG-HRP conjugate followed by calorimetric quantitation. (A) The labeling of VT680 to P-cadherin LP-DART had a DOL dependent effect on binding to human P-cadherin and Control LP-DART had no binding to human P-cadherin. (B) The labeling of VT680 to P-cadherin LP-DART and Control LP-DART affected binding to human CD3. P-cadherin LP-DART retained moderate binding even at a DOL of 2.0, whereas Control LP-DART lost its binding to human CD3 protein. (C) CTL Assay: Firefly luciferase expressing HCT116 cells and expanded human CD3+ T lymphocytes were co-incubated with increasing concentrations of P-cadherin LP-DART with different DOL of VT680. After $24 \mathrm{hr}$ the remaining viable cells were quantified by measuring the luciferase activity. The relative cytotoxicity observed at various concentrations of P-cadherin LP-DART-VT680 was plotted against the PBS treated samples. VT680 conjugation decreased the cytotoxic ability in a DOL dependent manner. 


\section{Ex vivo quantification of P-cadherin LP-DART in tumor and other organs}

FMT quantification of whole-body represents signal from accumulation of labeled reagent (probe) in tumor and other tissues in the torso region, as well as signal due to the probe in the circulation. To eliminate the residual signal contributed from the probe in circulation/ vasculature, we perfused the whole-body with saline and quantified the tumor and selected tissues (brain, heart, lungs, kidneys, liver and spleen) ex vivo by FMT at 48 , 96 and $240 \mathrm{hr}$ (Figure 4A-4C and Table 1). Liver was the main clearance site for both the P-cadherin LP-DART and Control LP-DART (Figure 4A and 4B). The kidneys and lungs also showed minor accumulation (Figure 4A), albeit at very low levels compared to liver. There was no considerable signal observed in other organs that were quantitated ex vivo. The comparison of quantitative values suggested that both P-cadherin LP-DART and Control LP-DART accumulated at similar levels in all the organs, except tumor. Quantitation of ex vivo FMT signal in tumor showed at least a 15-fold higher accumulation of P-cadherin LP-DART compared to Control LP-DART (29.9 pmol vs $2.31 \mathrm{pmol}$ at $48 \mathrm{hr}$ post-injection) (Figure $4 \mathrm{C}$ and Table 1). Although there was a trend towards decrease in tumor quantities between the 48,96 and $240 \mathrm{hr}$ time-points $(29.9,25.64$ and $21.28 \mathrm{pmol})$ in the P-cadherin LP-DART group, they were not statistically different. No detectable signal was observed in the ex vivo tumors of Control LP-DART at 96 and $240 \mathrm{hr}$ time-points (Figure 4C). Overall ex vivo FMT imaging confirmed the specific accumulation of P-cadherin LP-DART in the tumors as observed by in vivo imaging. FMT data also showed that liver was acting as a sink and the major site for clearance of both P-cadherin LP-DART and Control LP-DART (Table 1).

\section{Mechanistic characterization of P-cadherin LP-DART induced in vivo $\mathrm{T}$ cell trafficking to tumors in HCT-116 xenograft model}

P-cadherin LP-DART is a CD3 bispecific antibody that can mechanistically recruit effector $\mathrm{T}$ cells to tumors and trigger anti-tumor cytotoxic response. Previously, we have shown that P-cadherin LP-DART can regress HCT116 tumor xenografts [16]. Here we used FMT imaging to study T cell trafficking to tumors in HCT116 xenograft model, upon treatment with P-cadherin LP-DART. T cells were labeled with a membrane labeling NIR fluorophore CellVue ${ }^{\circledR}$ NIR815 (here after referred as CV815). To assess if CV815 labeling of $\mathrm{T}$ cells affected its survival or function, we performed in vitro $\mathrm{T}$ cell expansion and cytotoxicity assays. CV815-T cells and unlabeled T cells were stimulated with CD3/CD28 for $48 \mathrm{hr}$ and assessed for expansion. Unstimulated cells in the media increased by $\sim 2-3$ fold, whereas the stimulated $\mathrm{T}$ cells were increased by 4-5 fold, relative to number of cells seeded (Figure 5A). The CV815 labeled $\mathrm{T}$ cell expansion was greater than unlabeled T cells. The cytotoxic activity of CV815 labeled $\mathrm{T}$ cells and unlabeled $\mathrm{T}$ cells was evaluated by incubating with luciferase expressing HCT116 cells at different effector: target cell (E: T) ratios (20:1 to 0.312:1). Both CV815 labeled and unlabeled T cells showed similar cytotoxic potential at various E: $T$ ratios tested (Figure 5B). These results suggested that CV815 labeling did not affect the $\mathrm{T}$ cell function and hence were optimal for use in in vivo FMT imaging.

To determine the biodistribution of CV815-T cells in vivo and to select the concentration for $\mathrm{T}$ cell trafficking study, we performed a pilot study in naïve NSG mice. Different concentrations $\left(2-10 \times 10^{6}\right.$ cells $)$ of stimulated CV815-labeled $T$ cells were injected and imaged $24 \mathrm{~h}$ postengraftment (Supplementary Figure 3). The T cells were primarily localized in spleen and liver in a concentration dependent fashion. We determined that the mice injected with $5 \times 10^{6}$ cells provided signal above background and was not saturated in the major organs, spleen and liver. To assess P-cadherin LP-DART mediated T cell trafficking to tumors we adoptively transferred $5 \times 10^{6} \mathrm{CV} 815$ - $\mathrm{T}$ cells to HCT116 xenograft bearing mice. Mice with tumors $\left(\sim 300 \mathrm{~mm}^{3}\right)$ were randomized to treatment groups. Each group of animals received either unlabeled Control LPDART; unlabeled P-cadherin LP-DART subcutaneously (SQ) on day 0. After $24 \mathrm{hr}$ (day 1), CV815-T cells were injected intravenously (IV) and imaged by FMT longitudinally up to day 10 (Figure 6A). FMT imaging was performed 5 min after CV815-T-cell injection and the whole-body fluorescence signal was normalized as $100 \%$ injected dose. There was a decrease in tumor CV815 signal on all days compared to day $1(\sim 1.1 \%$ ID) in the Control LP-DART treated group (Figure 6B). The tumor CV815 signal in P-cadherin LP-DART treated group stayed relatively similar up to day 5 compared to day 1 $(\sim 1.1 \% \mathrm{ID})$. On day 8 there was an increase CV815 signal in tumors $(\sim 3.25 \% \mathrm{ID})$ suggesting increased infiltration $\mathrm{T}$ cells (Figure 6B). There was a 12-13 fold increase in CV815 signal in P-cadherin LP-DART group relative to the Control LP-DART group ( 3.25 vs $0.27 \%$ ID) on day 8 (Figure 6B) illustrating bispecific antibody mediated T cell trafficking to tumors (Figure 6C). On day 10 there was a significant decrease in tumor CV815-T cell signal which may be due to dilution of fluorescence due to $\mathrm{T}$ cell proliferation.

FMT4000 can image multiple near-infrared fluorescence channels without requiring spectral unmixing, thus we tried to visualize tumor specific co-localization of the P-cadherin LP-DART-VT680 and CV815-T cells. When the tumors were $\sim 300 \mathrm{~mm} 3 \mathrm{P}$-cadherin LP-DARTVT680 was injected via subcutaneous route and $24 \mathrm{~h}$ later the CV815-T cells were injected via intravenous route. Figure 6D shows the pseudo-colored tumor ROI for P-cadherin LP-DART-VT680 (red) and CV815-T 

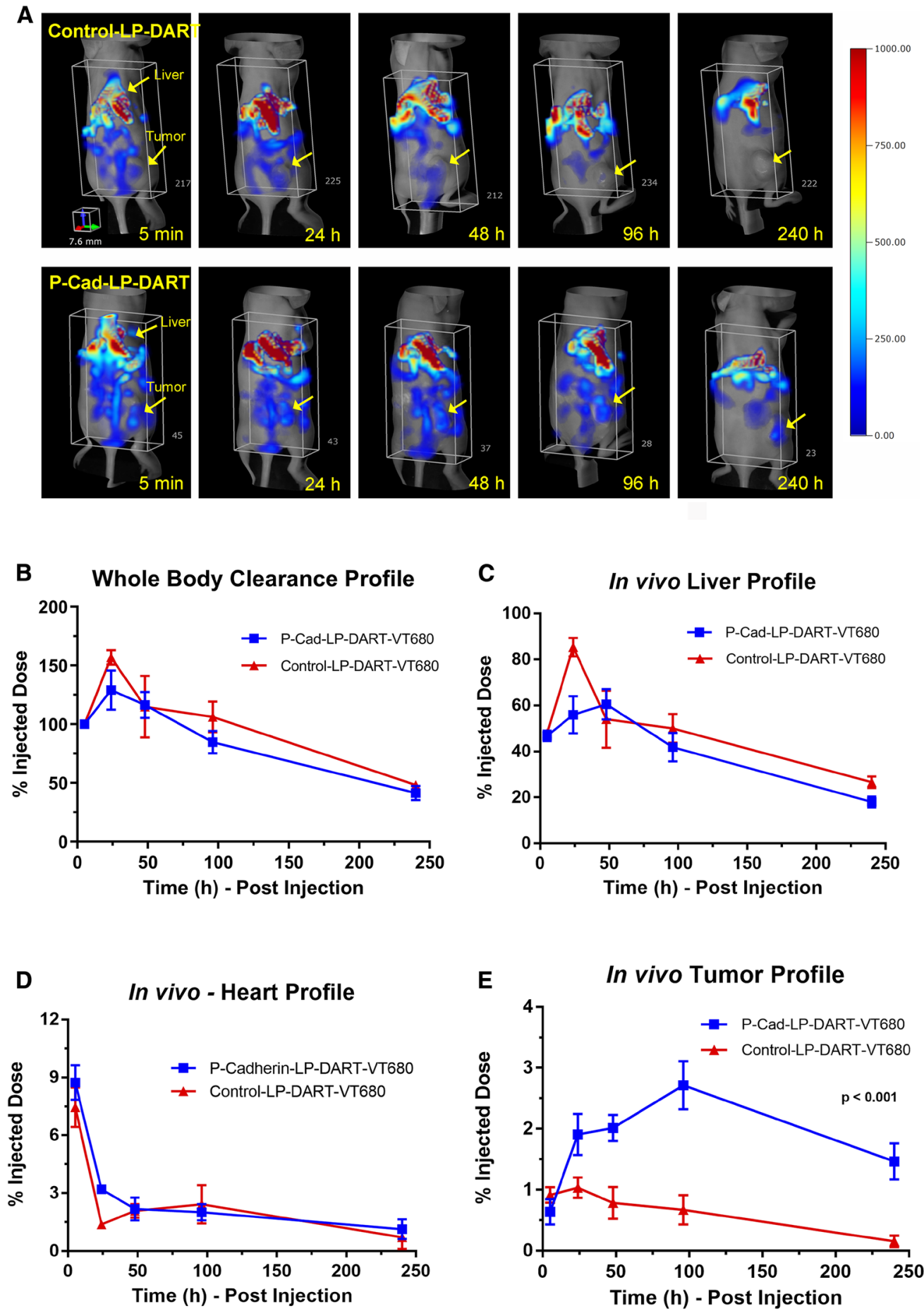

Figure 3: In vivo biodistribution and tumor targeting profile of P-cadherin LP-DART-VT680 and Control LP-DARTVT680. Female nu/nu mice bearing HCT116 xenografts were injected intravenously with P-cadherin LP-DART-VT680 and Control LP-DART-VT680 at doses equivalent to $1 \mathrm{nmol}$ of VT680 $(1.4 \mathrm{mg} / \mathrm{kg})$. FMT imaging was performed using the 680 laser at $5 \mathrm{~min}$, 24, 48, 96 and $240 \mathrm{hr}$ post-injection. (A) Representative 3-dimentional FMT images showing the whole-body (torso) biodistribution of P-cadherin LP-DART-VT680 and Control LP-DART-VT680. Arrows indicate the location of tumor and liver. (B) Relative whole-body profile of P-cadherin LP-DART-VT680 and Control LP-DART-VT680. (C) Relative liver profile of P-cadherin LP-DART-VT680 and Control LP-DART-VT680. (D) Upper torso was imaged separately to image heart and brain region. Relative heart profile of P-cadherin LP-DART-VT680 and Control LP-DART-VT680 shows a biphasic clearance profile. (E) Relative tumor accumulation of P-cadherin LPDART-VT680 and Control LP-DART-VT680. No major difference was observed between P-cadherin LP-DART-VT680 and Control LPDART-VT680 in the profiles of whole-body, liver, and heart. Tumor profile showed a significantly increased accumulation of P-cadherin LP-DART-VT680 compared to Control LP-DART-VT680. $n=4-6 /$ group; \pm SEM is represented in the graphs; Tumor accumulation: $p$ value $<0.001$, 2-way ANOVA. 
cells (green) and its overlay (yellow) on day 5 and day 8. There was no difference in the P-cadherin LP-DARTVT680 signal between day 5 and day 8, but an increase in CV815 signal in the tumors was visualized. Additionally, since we administered P-cadherin LP-DART-VT680 subcutaneously in this experiment we compared the tumor targeting profile with that of intravenous administration (Figure 3E) in the biodistribution study. An increased and sustained tumor targeting of P-cadherin LP-DART was observed when administered subcutaneously (peak on day 8 with $\sim 4.25 \%$ ID) compared to the intravenous route (peak on day 4 with $\sim 2.71 \% \mathrm{ID}$ ) (Figure $6 \mathrm{E}$ ). This suggests that subcutaneous injection may act as a depot and may provide a potential advantage (Figure 6E).

\section{DISCUSSION}

Molecular imaging like FMT and positron emission tomography (PET) are excellent non-invasive tools to evaluate the three pillars in drug discovery and development: exposure at the target site (Pillar 1), binding to the target (Pillar 2) and pharmacodynamic modulation (Pillar 3) [19, 20]. Imaging endpoints have important translational relevance as many of the endpoints can be adopted in clinical evaluation [20]. Here we have utilized FMT imaging to demonstrate the biodistribution and tumor targeting of P-cadherin LP-DART; the pharmacodynamic effect, i.e., the trafficking of $\mathrm{T}$ cells to the tumor microenvironment; the exposure profile of P-cadherin LP-DART, its scDb form (with no Fc domain) and the differences between administering P-cadherin LP-DART by subcutaneous vs intravenous route. Additionally, we harnessed the multi-channel capability of FMT imaging and examined the dynamics of targeting P-cadherin LPDART and T cell to the tumors.

Innovation in protein engineering has resulted in a variety of bispecific formats for cancer immunotherapy. Among these different bispecific platforms are the two drugs tested in patients: Catumaxomab and Blinatumomab [2, 7]. Catumaxomab is a $150 \mathrm{kDa}$ hybrid
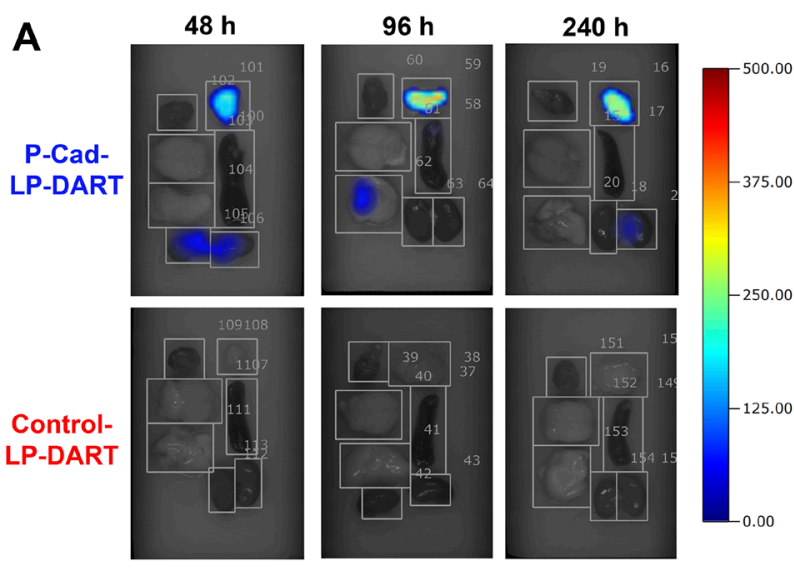

B

Ex-Vivo Liver Quantitation

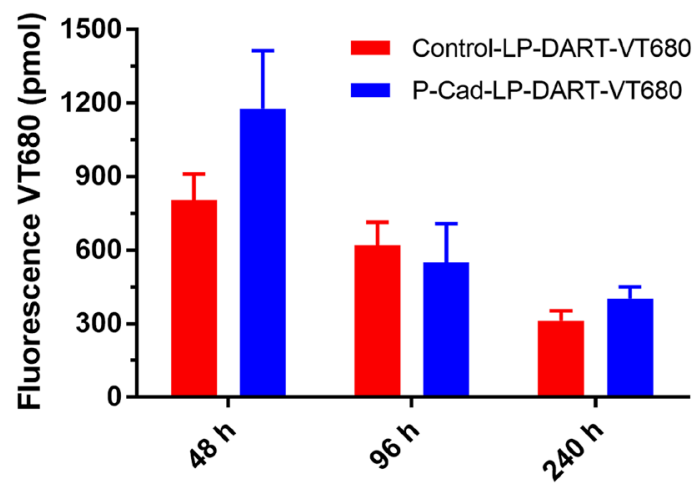

Hours after injection

C
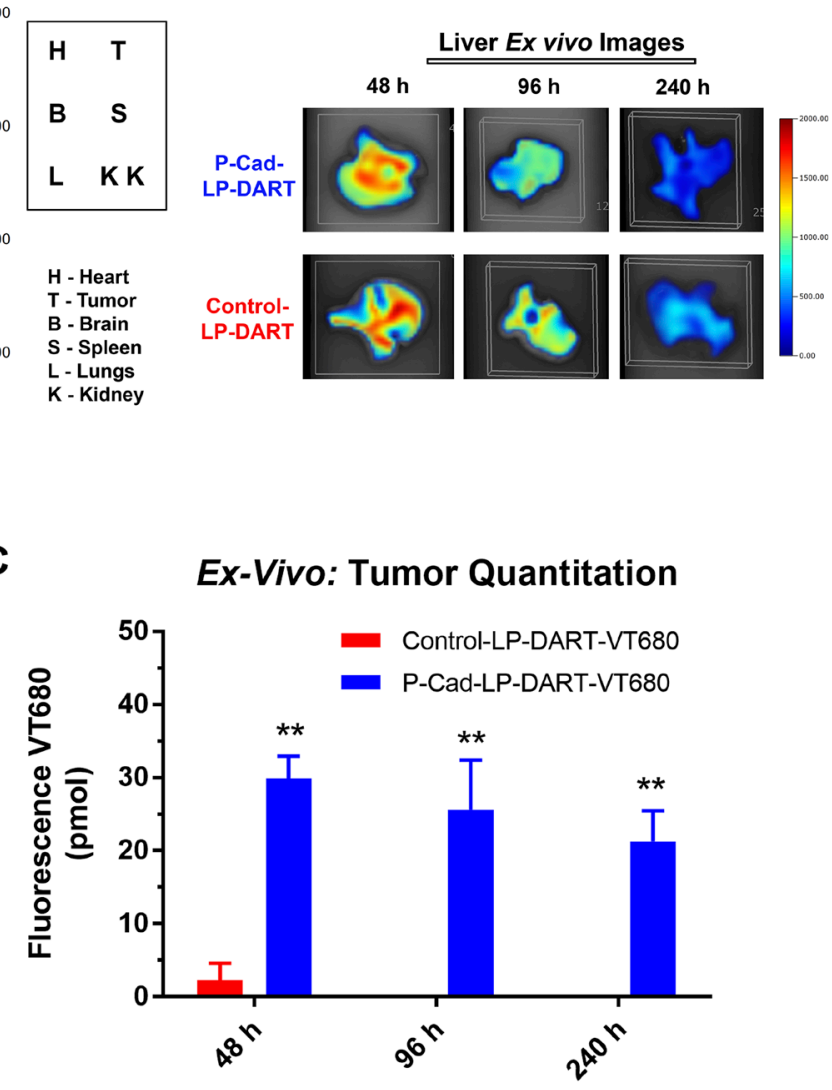

Hours - Post Injection

Figure 4: Ex vivo imaging and quantitation of accumulation in tumor and other organs. (A) Ex vivo FMT imaging panel showing the accumulation of P-cadherin LP-DART-VT680 and Control LP-DART-VT680 at 48, 96 and 240 hr in various organs: Tumor (T), Heart (H), Brain (B), Spleen (S), Lungs (L), Kidneys (K). No notable signal observed from any organs in this image except for tumor. Liver was imaged as a separate panel due to its size and was quantified. (B) Ex vivo quantitation of liver: No significant differences in liver was observed between P-cadherin LP-DART-VT680 and Control LP-DART-VT680 at 48, 96 and 240 hr. (C) Ex vivo quantitation of tumor showed significantly increased accumulation of P-cadherin LP-DART-VT680 compared to Control LP-DART-VT680 at 48,96 and 240 hr. $n=3-4 /$ group; $\pm \mathrm{SEM}$ is represented in the graphs; ${ }^{* *}=p$ value $<0.01$. 
Table 1: Ex vivo FMT quantitation of P-cadherin LP-DART and control LP-DART accumulation in tumor and selected organs

\begin{tabular}{lccccccc}
\hline \multicolumn{2}{l}{ P-cadherin LP-DART } & \multicolumn{7}{l}{} \\
\hline Time & Tumor & Liver & Brain & Heart & Kidneys & Lungs & Spleen \\
\hline $\mathbf{4 8} \mathbf{~ h}$ & $29.90^{* *}$ & 1177.12 & 0.29 & 0.00 & 9.51 & 11.13 & $0.47^{* *}$ \\
$\mathbf{9 6} \mathbf{~ h}$ & $25.61^{*}$ & 549.29 & 0.63 & 0.87 & 18.26 & 9.49 & $0.66^{* *}$ \\
$\mathbf{2 4 0} \mathbf{~ h}$ & $21.29^{* *}$ & 402.52 & 0.04 & 0.24 & 5.86 & 0.01 & 0.09 \\
\hline
\end{tabular}

\section{Control LP-DART}

\begin{tabular}{lccccccc}
\hline Time & Tumor & Liver & Brain & Heart & Kidneys & Lungs & Spleen \\
\hline $\mathbf{4 8} \mathbf{~ h}$ & 2.31 & 805.33 & 0.08 & 0.06 & 6.46 & 1.48 & 0.05 \\
$\mathbf{9 6} \mathbf{~ h}$ & 0.00 & 619.42 & 0.07 & 0.33 & 30.93 & 0.02 & 0.07 \\
$\mathbf{2 4 0} \mathbf{~ h}$ & 0.00 & 313.05 & 0.00 & 0.00 & 4.82 & 0.00 & 0.00 \\
\hline
\end{tabular}

Significantly higher accumulation of P-cadherin LP-DART was observed in tumor, compared to control LP-DART at all three ex vivo time points, $48 \mathrm{~h}, 96 \mathrm{~h}$ and $240 \mathrm{~h}$. No significant difference between P-cadherin LP-DART and control LP-DART was observed in liver, brian, heart, kidneys and lungs. Although spleen showed significantly higher accumulation of P-cadherin LP-DART compared to control LP-DART at $48 \mathrm{~h}$ and $96 \mathrm{~h}$, the quantities were very low.

IgG2 like molecule targeting epithelial cell adhesion molecule (EpCAM), a tumor antigen and CD3 on the $\mathrm{T}$ cells in contrast to Blinatumomab, which is a $55 \mathrm{kDa}$ bispecific $\mathrm{T}$ cell engager (BiTE) targeting CD19 on the cancer cell and CD3 on the T cell. The pharmacokinetics of BiTE class of therapeutic is very interesting and is a differentiating property. $\mathrm{T}$ cell bispecifics possessing a full-length $\mathrm{IgG}$ Fc region demonstrate a longer halflife (2.13 days) as was observed in Catumaxomab. The incorporation of an Fc fragment increases the molecular weight and the circulation half-life through its interactions with the neonatal Fc receptor (FcRn) [21]. Blinatumomab, which lacks an Fc region and has only the single chain variable fragments ( $\mathrm{scFv}$ ) targeting CD19 and CD3 demonstrated a half-life of $2 \mathrm{hr}[2,7]$. In our mouse studies using imaging-based approach we showed accelerated clearance to the P-cadherin DART ( $\mathrm{scDb}$ ) compared to the P-cadherin LP-DART, which has an engineered Fc region in the molecule. The halflife of P-cadherin LP-DART in a human FcRn transgenic mouse model was determined to be $\sim 4.4$ days [10]. Similar to our observation by FMT, PET imaging of ${ }^{89} \mathrm{Zr}$ AMG211, a BiTE molecule targeting carcinoembryonic antigen (CEA) and CD3e showed very rapid clearance through kidneys [22]. In this study the plasma halflife of ${ }^{89} \mathrm{Zr}-\mathrm{AMG} 211$ was determined to be $\sim 1 \mathrm{hr}$. Such
A

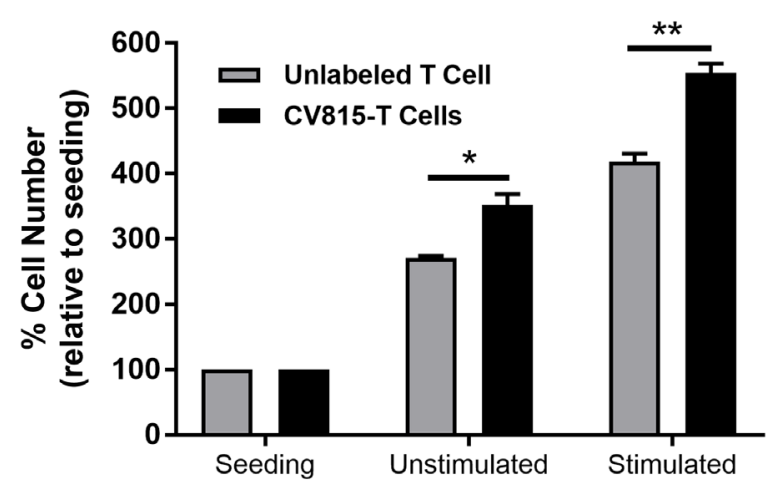

B

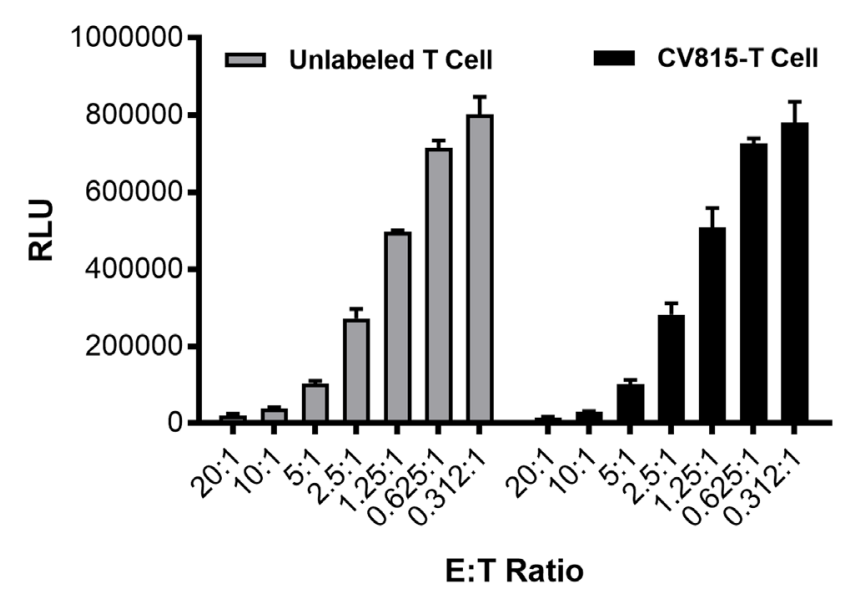

Figure 5: Effect of in vitro labeling of CD3+ T cells with CellVue ${ }^{\circledR}$ NIR815 fluorophore. (A). In vitro T Cell expansion of CV815 labeled CD3+ T cells. Unlabeled and CV815-T cells were incubated under unstimulated or CD3/CD28 stimulation condition for 48 $\mathrm{hr}$ and quantified the number of cells using CellTiter-Glo ${ }^{\circledR}$ Luminescent Cell Viability kit. (B) In vitro CTL assay was performed by mixing different E: T ratios with 20 pM P-cadherin LP-DART for $24 \mathrm{hr}$. The number of viable target cells was estimated using ONE-Glo ${ }^{\circledR}$ luciferase kit. $\pm \mathrm{SEM}$ is represented in the graphs; ${ }^{*}=p$ value $<0.05 ;{ }^{* *}=p$ value $<0.01$. 
A

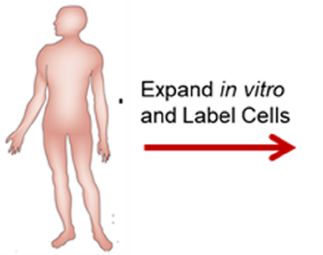

Isolate from Healthy Volunteer

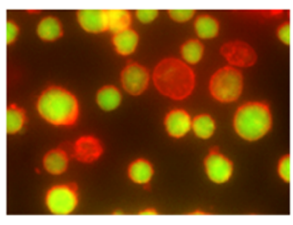

Cells Labeled with CV815 Quality Control Tests
B

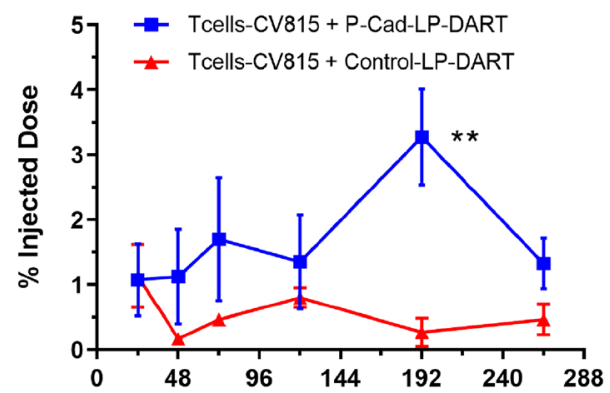

Time (h) - Post Injection of P-Cad-LP-DART

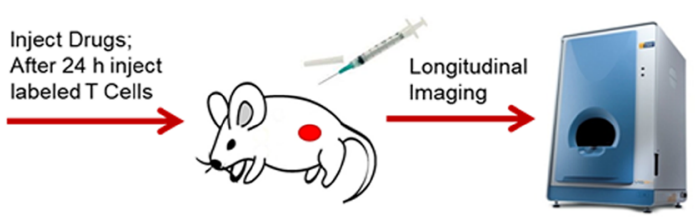

Tumor Bearing Mice
Non-Invasive FMT Imaging

\section{T Cell-CV815 in Tumors on Day 8}
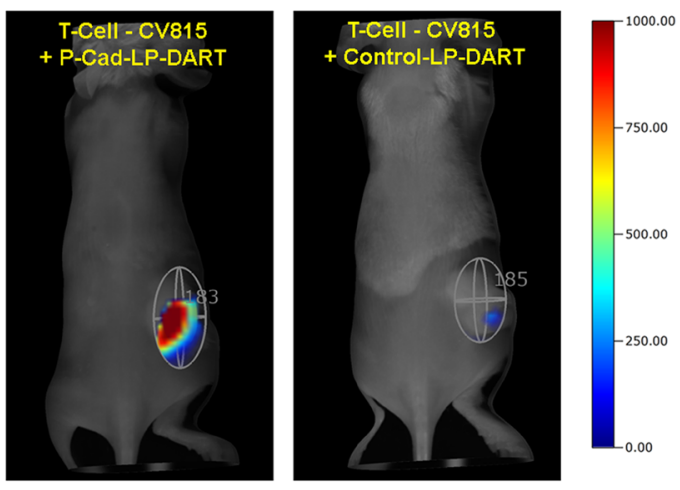

\section{E P-cadherin-LP-DART (SQ vs IV):} In vivo Accumulation in tumors

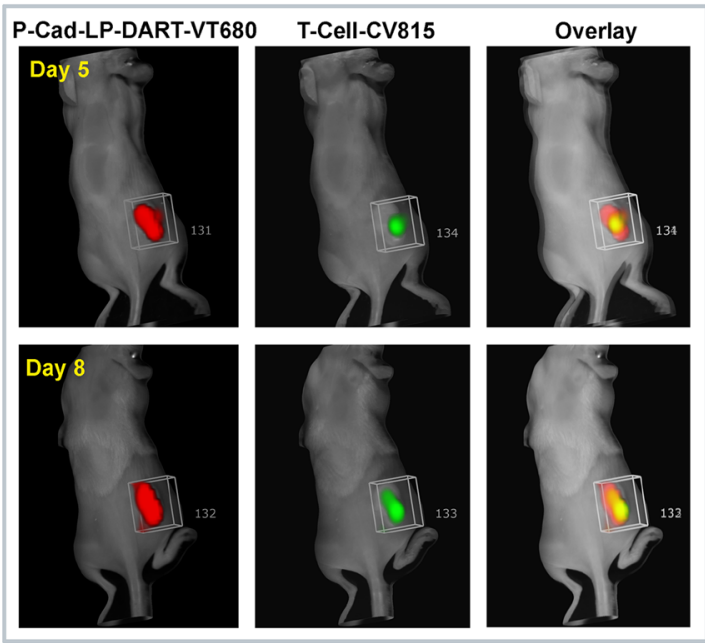

Time (h) - Post Injection of P-Cad-LP-DART

Figure 6: In vivo T cell trafficking in HCT-116 xenograft model by FMT imaging. Female NSG mice bearing HCT116 xenografts were injected subcutaneously with P-cadherin LP-DART, P-cadherin LP-DART-VT680 or Control LP-DART at 2 mg/kg dose. After $24 \mathrm{hr}$ the CV815-T cells $\left(5 \times 10^{6}\right.$ cells $)$ were injected intravenously. FMT imaging was performed longitudinally using the 680 $\mathrm{nm}$ laser for VT680 and the $800 \mathrm{~nm}$ laser for CV815. (A) Schematic showcasing the design of T cell trafficking study. (B) Profile of in vivo tumor trafficking of CV815-T-Cells in groups treated with unlabeled P-cadherin LP-DART and Control LP-DART. No significant difference of CV815-T-Cells was observed in until $120 \mathrm{hr}$ post-injection. At $192 \mathrm{hr}$ there was a significant increase in CV815 signal in tumors $\left(n=3-4\right.$ /group; \pm SEM is represented at all time points; $t$-test, ${ }^{* *} p$-value $\left.<0.01\right)$. (C) FMT images on day 8 (192 hr) showing the CV815-T cells in tumor of mice treated with unlabeled P-cadherin LP-DART whereas minimal signal was observed in Control LP-DART treated mice. (D) Representative FMT images on day 5 and 8 from a separate cohort of animals showing co-localization of P-cadherin LPDART-VT680 (red) with CV815-T cells (green). (E) Comparison of profiles of tumor accumulation when P-cadherin LP-DART-VT680 was administered via intravenous route (IV) and subcutaneous route (SQ). FMT profile shows a slower, sustained and a slightly increased P-cadherin LP-DART-VT680 accumulation by subcutaneous administration. 
short plasma half-life requires continuous infusion and frequent administration of the drug, compared to weekly or bi-weekly administration of Fc-containing bispecific molecules [23, 24]. We also compared the exposure of P-cadherin LP-DART when administered intravenously or subcutaneously using FMT imaging. We saw an extended half-life with increased exposure of P-cadherin LP-DART at the tumor site by subcutaneous administration compared to intravenous route $(4.14 \% \mathrm{ID}$ on day $11 \mathrm{vs} 1.46 \% \mathrm{ID}$ on day 10$)$. Since we are quantifying the fluorophore signal as a measure of the drug, it would be interesting to know the contribution of the intact P-cadherin LP-DART to its catabolized antibody.

Previously we demonstrated that the pharmacology of P-cadherin LP-DART is dependent on the expression of P-cadherin on the tumor cells. This study was performed using the HCT116 cell line which has been shown to express high levels of P-cadherin (antigen-binding capacity of 114,000) [10]. Targeting of P-cadherin LPDART was dependent on the antigen specificity as the Control LP-DART which has similar affinity to T cells but does not bind to P-cadherin or the HCT116 cells, did not show any meaningful tumor accumulation. We did not observe a major difference in tumor targeting of $\mathrm{P}$-cadherin LP-DART in the presence or absence of immune cells (Supplementary Figure 4A and 4B) suggesting that the tumor targeting was primarily dependent on the tumor antigen. We did not see significant differences between levels of Control LP-DART and P-cadherin LP-DART in any other tissues as the molecules bind specifically to human epitopes. Evaluating the biodistribution using a mouse reactive P-cadherin LP-DART or human CD3 expressing transgenic mouse could provide further insight into this finding. Biodistribution studies with labeled molecules have shown increased distribution of $\mathrm{CD} 3$ bispecifics to $\mathrm{T}$ cell rich tissues such as the lymph node and spleen in human CD3e transgenic mouse model. Accumulation in normal tissues like liver and spleen may act as a sink and impact the plasma and tumor exposure [25]. Such labeled reagents and tools provide very valuable information on the on- and off-target binding both in pre-clinical models and in patients. It may also provide information on potential target-mediated safety concerns that may be anticipated in patients and help in planning the mitigation strategy [20, 26, 27].

The pharmacology of the bispecific antibody is elicited by forming a bridge between the target (i.e., tumor) cell and the effector ( $\mathrm{T}$ cell) cell [28]. In order to demonstrate the mechanism of P-cadherin LP-DART we imaged of both drug and the $\mathrm{T}$ cell simultaneously. Advances in immuno-therapy, along with the interest in tracking immune cells in vivo in patients and in pre-clinical models, has resulted in the development of multiple molecular imaging methodologies [29]. Among them optical imaging, magnetic resonance imaging (MRI) and PET have emerged as major players. In this study we took advantage of the multi-channel capability of FMT imaging and narrow fluorescence spectrums of near-infrared fluorophores which result in minimum contribution across channels [18, 20]. We labeled the T cells with CV815, a lipophilic dye which has a polar head group and an aliphatic hydrocarbon chain. The aliphatic chain gets noncovalently incorporated into the lipid bilayer of the cells. To our knowledge this is the first study to demonstarte non-invasively the dynamics and simultaneous trafficking of $\mathrm{T}$ cells and a bispecific antibody to tumors. Similar to our previous observation by immunohistochemistry [16], we saw significantly higher number of $\mathrm{T}$ cells in the tumors (Figure 6C). Although CV815 based T cell tracking has several advantages, it may underestimate the number of $\mathrm{T}$ cells since the fluorescence is diluted by one-half at each subsequent division of the proliferating $\mathrm{T}$ cell. Additionally, since optical imaging has not yet been adopted as a non-invasive modality in clinic, its application is limited to mechanistic studies in pre-clinical models. It will be interesting to evaluate the trafficking of T cells using newer, potentially translational, PET imaging based immuno-oncology biomarkers like IL-2, Granzyme $\mathrm{B}$ and CD8-minibody [30-33]. In summary, using FMT imaging we have shown the targeting of P-cadherin LP-DART (Pillar 1 and 2) and the trafficking of T cells (Pillar 3) to the tumor microenvironment in pre-clinical models. P-cadherin LP-DART (PF-06671008) is currently in Phase 1 trials evaluating safety, pharmacokinetics and pharmacodynamics of increasing doses of PF-06671008 in patients with advanced solid tumors. This study has great translational relevance and can aid as a proof of principle for the use of molecular imaging in patients to assess three pillars.

\section{MATERIALS AND METHODS}

\section{Cell culture}

HCT116 (ATCC ${ }^{\circledR}$ CCL-247TM) cells line were purchased from American Type Culture Collection (ATCC) and cultured as per their instructions. Cell lines were authenticated, and pathogen tested by IDEXX Bioresearch prior to in vivo studies.

\section{Bispecific antibodies and fluorophore labeling}

The bispecific molecules used in this study were produced at Pfizer Inc. The bispecific antibodies P-cadherin DART, a single chain diabody $(\mathrm{scDb})$ (mol. wt. of $\sim 50 \mathrm{kDa}$ ), P-cadherin LP-DART (with mol. wt. of $\sim 100 \mathrm{kDa}$ ) (Figure 1A-1D) and Control LP-DART (with mol. wt. of $\sim 100 \mathrm{kDa}$ ) were labeled with VT680 using a VivoTag ${ }^{\circledR} 680 \mathrm{XL}$ protein labeling kit as per the manufacturer's instructions (Perkin Elmer Inc) and has been previously described [17]. Briefly, the VT680 has a succinimidyl ester group, which reacts with the primary 
amine group on the bispecific antibodies to form a stable amide linkage. The reactions were carried out in PBS and after conjugation reaction the free fluorophore was removed using a protein purification column. Degree of labeling (DOL) of VT680 conjugation to bispecific antibodies was evaluated by measuring the absorbance at $280 \mathrm{~nm}$ and $668 \mathrm{~nm}$ using a NanoDrop ${ }^{\text {TM }} 8000$ (Thermo Fisher Scientific). Reagents with DOL between 1-3 VT680 molecules/protein were used in this study.

\section{Isolation PBMNCs and expansion of human CD3+ lymphocytes}

Whole blood in EDTA was freshly isolated from healthy human donors who were provided with informed consent. Peripheral blood mononuclear cells (PBMNCs) were isolated by density gradient centrifugation layered over Histopaque ${ }^{\circledR} 1077$ in ACCUSPINTM conical tubes (Sigma-Aldrich) following the manufacturer's protocol. PBMNCs were washed twice with Dulbecco's phosphate buffered saline (DPBS) containing $2 \mathrm{mM}$ EDTA and subjected to further fractionation by negative magnetic bead selection to isolate CD3+ lymphocytes using a human CD3+ negative isolation kit (Stem Cell Technologies). CD3+ lymphocytes were resuspended with CTS ${ }^{\mathrm{TM}}$ OpTmizer ${ }^{\mathrm{TM}}$ T-Cell Expansion Serum-Free Media supplemented with $2 \times$ GlutaMax-1, 1\% PenStrep and $20 \mathrm{ng} / \mathrm{mL}$ recombinant human IL-2 (Thermo Fisher Scientific). Dynabeads ${ }^{\text {TM }}$ Human T-Expander CD3/ CD28 magnetic beads (Thermo Fisher Scientific) were added to T-cells at a ratio of 2 beads per cell and cultured for one week. At the time of harvest, beads were removed with a magnet and cells were resuspended in DPBS for in vivo inoculation or in Recovery ${ }^{\mathrm{TM}}$ Cell Culture Freezing Medium (Thermo Fisher Scientific) for cryopreservation.

\section{T cell labeling with CellVue ${ }^{\mathrm{TM}}$ NIR815 fluorophore}

$\mathrm{T}$ cells were labeled with CellVue ${ }^{\circledR}$ NIR815 fluorophore (here-after referred as CV815) using CellVue $^{\circledR}$ NIR815 Fluorescent Cell Labeling Kit as per the manufacturer's instructions (Thermo Fisher Scientific). T cells $\left(1 \times 10^{7}\right.$ cells $\left./ \mathrm{mL}\right)$ were labeled at the final dye concentration of $2 \mu \mathrm{M}$ in serum free medium for $3 \mathrm{~min}$ and the residual dye after reaction was quenched by serum. After labeling the viability of T cells was evaluated and reactions with viability $>95 \%$ were used for the in vitro functional assays and in vivo studies. To evaluate the T cell expansion in vitro CV815-T cells were incubated in CTS ${ }^{\mathrm{TM}}$ OpTmizer ${ }^{\mathrm{TM}}$ T-Cell Expansion Serum-Free Media supplemented with $2 \times$ GlutaMax-1, $1 \%$ PenStrep and $20 \mathrm{ng} / \mathrm{mL}$ recombinant human IL-2. For stimulation the Dynabeads ${ }^{\mathrm{TM}}$ Human T-Expander CD3/CD28 magnetic beads were added to T-cells at a ratio of 2 beads per cell and cultured for $48 \mathrm{hr}$. The number of viable cells were quantified using CellTiterGlo $^{\circledR}$ Luminescent Cell Viability Assay (Promega Life Sciences). To assess the effect of CV815 labeling on cytotoxic activity of CD3+ T cells firefly luciferasetransfected HCT116 cells (T) that endogenously expressed P-cadherin were mixed with human CD3+ lymphocytes (E) at different $\mathrm{E}: \mathrm{T}$ ratios (20:1 to $0.312: 1)$ with $20 \mathrm{pM}$ P-cadherin LP-DART. After 24 $\mathrm{h}$ of incubation at $37^{\circ} \mathrm{C}$ under $5 \% \mathrm{CO}_{2}$ atmosphere the remaining luciferase activity from the viable tumor target cells was assessed by addition of an equal volume ONE$\mathrm{Glo}^{\mathrm{TM}}$ luciferase assay reagent (Promega Life Sciences).

\section{P-cadherin and CD3 binding ELISA}

The binding of VT680 labeled bispecific antibodies to soluble P-cadherin and CD3 proteins were evaluated by ELISA. The soluble P-cadherin and CD3 proteins were expressed using Pfizer's hu-CD3 epsilon-delta his8 and cyno-P-cadherin-his6 constructs as described previously [10]. ELISA plates were coated overnight with $1 \mu \mathrm{g} / \mathrm{mL}$ huCD3 or cyno-P-cadherin soluble proteins followed by incubation of test samples serially diluted in blocking buffer (3\% milk in PBS) for $1 \mathrm{hr}$ at room temperature. Plates were washed with PBS and bound proteins were detected using anti-human IgG-HRP diluted in blocking buffer. Plates were washed, and the bound complexes were detected with TMB substrate. Reaction was stopped with $\mathrm{H}_{2} \mathrm{SO}_{4}$ and the absorbance was measured at $450 \mathrm{~nm}$. Each concentration was evaluated in triplicates. EC50 was determined by non-linear curve fit of relative absorbance using GraphPad Prism 7.04 software (GraphPad Software).

\section{Cytotoxic T lymphocyte (CTL) activity}

CTL assay was performed as described before [16]. Briefly, firefly luciferase transfected HCT116 cells (T) that endogenously expressed P-cadherin were mixed with human $\mathrm{CD} 3+$ lymphocytes $(\mathrm{E})$ at the ratio of 5:1, E: T. VT680 labeled P-cadherin LP-DART, P-cadherin DART (scDb) and Control LP-DART bispecific antibodies were added at serial dilutions and incubated for $24 \mathrm{hr}$ at $37^{\circ} \mathrm{C}$ under $5 \% \mathrm{CO}_{2}$ atmosphere. The remaining luciferase activity from the viable tumor target cells after $24 \mathrm{hr}$ was assessed by addition of an equal volume ONE-Glo ${ }^{\mathrm{TM}}$ luciferase assay reagent (Promega Life Sciences). Relative light units (RLU) were collected with an Envision plate reader (Perkin Elmer Inc.) as counts per second (cps). Percent cytotoxicity was determined by the following formula, 100-100*(RLU value of test samples/RLU of control samples). Each concentration was evaluated in triplicate. EC50 was determined by non-linear curve fit of percent cytotoxicity using GraphPad Prism 7.04 software (GraphPad Software). 


\section{In vivo biodistribution study}

All animal studies were performed as per the animal use protocols approved by Pfizer Inc's Institutional Animal Care and Use Committee (IACUC) in an AAALAC accredited facility. Animals were housed under standard 12:12 light: dark cycle in individually ventilated cages at a room temp of $72^{\circ} \mathrm{F}( \pm 2 \mathrm{~F})$, relative humidity between 30 $70 \%$ and were provided with food and water, ad libitum.

Biodistribution of P-cadherin LP-DART was evaluated in a HCT116 subcutaneous xenograft model. HCT116 cells $\left(5 \times 10^{6}\right.$ cells/mouse $)$ mixed with $4 \mathrm{mg} /$ $\mathrm{mL}$ Cultre ${ }^{\circledR}$ basement membrane extract (Trevigen) were implanted s. c. on the dorsal right flank of 6-8-weekold NSG or nu/nu female mice purchased from The Jackson Laboratory or Charles River Laboratories. After implantation tumors were measured twice weekly by calipers. Imaging studies were initiated when the tumors were $\sim 300 \mathrm{~mm}^{3}$ in volume. The feed for the mice was changed to irradiated alfalfa free diet (AIN76A from Newco Distributors Inc.) 48-72 hr prior to the start of imaging. The animals were injected i. v or s. c. with probes normalized to $1 \mathrm{nmol}$ VT680 concentration (calculated based on DOL). Non-invasive FMT imaging (whole-body) was performed longitudinally post-injection of the probes at various time points, up to $240 \mathrm{hr}$. Animals were anesthetized with $2-3 \%$ isoflurane and maintained under anesthesia in a $37^{\circ} \mathrm{C}$ warm chamber during each imaging session. For ex vivo imaging time points representative animals from each group were perfused under isoflurane anesthesia with PBS/Saline to remove the blood. After perfusion and confirmation of euthanasia, tissues (tumor, liver, spleen, kidney, brain and lungs) were immediately collected for ex vivo FMT imaging. Quantitation of FMT data was done using TrueQuant ${ }^{\mathbb{B}}$ software (Perkin Elmer Inc). The fluorescence signal measured at $5 \mathrm{~min}$ post-injection was used to calculate the percent injected dose (100\%ID). Each imaging group had 3-6 animals for each time point.

\section{In vivo evaluation of $\mathrm{T}$ cell trafficking}

$\mathrm{T}$ cells were labeled with CV815 as mentioned before. A pilot in vivo study was performed in 6-8-weekold NSG mice with different concentrations of CV815-T cells $\left(1,5\right.$ and $10 \times 10^{6}$ cells) to determine the dose with optimal signal. To evaluate the trafficking of $\mathrm{T}$ cell to tumors upon treatment with P-cadherin LP-DART, we used the human $\mathrm{T}$ cell adoptive transfer model bearing HCT116 subcutaneous xenograft model. HCT116 cells were implanted in NSG mice as described above and study was initiated when the tumors were $\sim 300$ $\mathrm{mm}^{3}$. Animals were treated with P-cadherin LP-DART (Unlabeled or Labeled with VT680, dose $-2 \mathrm{mg} / \mathrm{kg}$ ) s. c. on day 0 . CV815-T cells $\left(5 \times 10^{6}\right.$ cells $/$ mice $)$ were injected i. v. $24 \mathrm{hr}$ post-injection of drugs (day 1). Multi- channel non-invasive FMT imaging was performed on day 0 (for P-cadherin LP-DART-VT680), day 1, 2, 3, 5, 8 and 11 (for CV815-T cells and P-cadherin LPDART-VT680, groups as applicable) post-treatment with P-cadherin LP-DART.

\section{Abbreviations}

3D: Three-dimensional; Ab: Antibody; ADCC: Antibody-dependent cellular cytotoxicity; CDC: Complement-dependent cytotoxicity; CTL: Cytotoxic T lymphocyte; CV815: CellVue ${ }^{\circledR}$ NIR815; DART: Dualaffinity re-targeting; DOL: Degree of labeling; EC50: Half maximal effective concentration; EFN: Effector function null mutations; EpCAM: Epithelial cell adhesion molecule; E:T ratios: Effector to target cell ratios; FcRn: Neonatal Fc receptor; FMT: Fluorescence molecular tomography; ID: Injected dose; MRI: Magnetic resonance imaging; NHS: N-hydroxy succinimidyl ester; NIR: Near-infrared; PBMNC: Peripheral blood mononuclear cell; PET: Positron emission tomography; RLU: Relative light unit; ROI: Region of Interest; scDb: Single-chain diabody; scFv: Single-chain variable fragment; TCR: T cell receptor; TMB - 3,3',5,5'-tetramethylbenzidine; VT680: VivoTag ${ }^{\circledR} 680$ XL.

\section{Author contributions}

Conception and design: VG; CM; AG. Development of methodology: VG; AR; TF; RN; AG. Acquisition of data (provided animals, acquired and managed patients, provided facilities, etc.): VG; AR; TF; JC; TC, RN; JD; AG. Analysis and interpretation of data (e.g., statistical analysis, biostatistics, computational analysis): VG; AR; TF; CM; AG. Writing, review, and/or revision of the manuscript: VG; AR; TM; JD; AG.

\section{ACKNOWLEDGMENTS}

We thank the in vivo Pharmacology team (Oncology Research and Development, Pfizer Inc., CA, USA) and Comparative Medicine technical staff for their help in the in vivo studies and Donna M Zyry (Comparative Medicine, Pfizer Inc.) for editing the manuscript. P-cadherin LP-DART was generated as a part of Pfizer Inc.,/MacroGenics, Inc. collaboration. This project was funded by Pfizer Inc. Note: DART $^{\circledR}$ is a registered trademark of MacroGenics, Inc.

\section{CONFLICTS OF INTEREST}

All authors are or were full time or part-time employees of Pfizer, Inc.

\section{FUNDING}

This project was funded by Pfizer, Inc. 


\section{REFERENCES}

1. Elgundi Z, Reslan M, Cruz E, Sifniotis V, Kayser V. The state-of-play and future of antibody therapeutics. Adv Drug Deliv Rev. 2017; 122:2-19. https://doi.org/10.1016/j. addr.2016.11.004. [PubMed]

2. Thakur A, Lum LG, Mittal S. Bispecific Antibody Armed T Cells to Target Cancer Cells. Methods Mol Biol. 2018; 1722:117-126. https://doi.org/10.1007/978-1-4939-75532 8. [PubMed]

3. Yang F, Wen W, Qin W. Bispecific Antibodies as a Development Platform for New Concepts and Treatment Strategies. Int J Mol Sci. 2016; 18:48. https://doi. org/10.3390/ijms18010048. [PubMed]

4. Fan G, Wang Z, Hao M, Li J. Bispecific antibodies and their applications. J Hematol Oncol. 2015; 8:130. https:// doi.org/10.1186/s13045-015-0227-0. [PubMed]

5. Baeuerle PA, Reinhardt C. Bispecific T-cell engaging antibodies for cancer therapy. Cancer Res. 2009; 69:49414944. https://doi.org/10.1158/0008-5472.CAN-09-0547. [PubMed]

6. Huehls AM, Coupet TA, Sentman CL. Bispecific T-cell engagers for cancer immunotherapy. Immunol Cell Biol. 2015; 93:290-296. https://doi.org/10.1038/icb.2014.93. [PubMed]

7. Krishnamurthy A, Jimeno A. Bispecific antibodies for cancer therapy: A review. Pharmacol Ther. 2018; 185:122134. https://doi.org/10.1016/j.pharmthera.2017.12.002. [PubMed]

8. May C, Sapra P, Gerber HP. Advances in bispecific biotherapeutics for the treatment of cancer. Biochem Pharmacol. 2012; 84:1105-1112. https://doi.org/10.1016/j. bcp.2012.07.011. [PubMed]

9. Moore PA, Zhang W, Rainey GJ, Burke S, Li H, Huang L, Gorlatov S, Veri MC, Aggarwal S, Yang Y, Shah K, Jin L, Zhang S, et al. Application of dual affinity retargeting molecules to achieve optimal redirected T-cell killing of B-cell lymphoma. Blood. 2011; 117:4542-4551. https:// doi.org/10.1182/blood-2010-09-306449. [PubMed]

10. Root AR, Cao W, Li B, LaPan P, Meade C, Sanford J, Jin M, O’Sullivan C, Cummins E, Lambert M, Sheehan AD, Ma W, Gatto S, et al. Development of PF-06671008, a Highly Potent Anti-P-cadherin/Anti-CD3 Bispecific DART Molecule with Extended Half-Life for the Treatment of Cancer. Antibodies (Basel). 2016; 5:6. https://doi. org/10.3390/antib5010006. [PubMed]

11. Atwell S, Ridgway JB, Wells JA, Carter P. Stable heterodimers from remodeling the domain interface of a homodimer using a phage display library. J Mol Biol. 1997; 270:26-35. https://doi.org/10.1006/jmbi.1997.1116. [PubMed]

12. Ridgway JB, Presta LG, Carter P. 'Knobs-into-holes' engineering of antibody $\mathrm{CH} 3$ domains for heavy chain heterodimerization. Protein Eng. 1996; 9:617-621. https:// doi.org/10.1093/protein/9.7.617. [PubMed]
13. Imai K, Hirata S, Irie A, Senju S, Ikuta Y, Yokomine K, Harao M, Inoue M, Tsunoda T, Nakatsuru S, Nakagawa $\mathrm{H}$, Nakamura Y, Baba H, Nishimura Y. Identification of a novel tumor-associated antigen, cadherin $3 / \mathrm{P}$-cadherin, as a possible target for immunotherapy of pancreatic, gastric, and colorectal cancers. Clin Cancer Res. 2008; 14:64876495. https://doi.org/10.1158/1078-0432.CCR-08-1086. [PubMed]

14. Shimoyama Y, Hirohashi S, Hirano S, Noguchi M, Shimosato Y, Takeichi M, Abe O. Cadherin cell-adhesion molecules in human epithelial tissues and carcinomas. Cancer Res. 1989; 49:2128-2133. [PubMed]

15. Peralta Soler A, Knudsen KA, Salazar H, Han AC, Keshgegian AA. P-cadherin expression in breast carcinoma indicates poor survival. Cancer. 1999; 86:1263-1272. $\quad$ https://doi.org/10.1002/ (SICI)1097-0142(19991001)86:7\%3C1263::AIDCNCR23\%3E3.0.CO;2-2. [PubMed]

16. Fisher TS, Hooper AT, Lucas J, Clark TH, Rohner AK, Peano B, Elliott MW, Tsaparikos K, Wang H, Golas J, Gavriil M, Haddish-Berhane N, Tchistiakova L, et al. A CD3-bispecific molecule targeting P-cadherin demonstrates $\mathrm{T}$ cell-mediated regression of established solid tumors in mice. Cancer Immunol Immunother. 2018; 67:247-259. https://doi.org/10.1007/s00262-017-2081-0. [PubMed]

17. Giddabasappa A, Gupta VR, Norberg R, Gupta P, Spilker ME, Wentland J, Rago B, Eswaraka J, Leal M, Sapra P. Biodistribution and Targeting of Anti-5T4 Antibody-Drug Conjugate Using Fluorescence Molecular Tomography. Mol Cancer Ther. 2016; 15:2530-2540. https://doi. org/10.1158/1535-7163.MCT-15-1012. [PubMed]

18. Vasquez KO, Casavant C, Peterson JD. Quantitative whole body biodistribution of fluorescent-labeled agents by noninvasive tomographic imaging. PLoS One. 2011; 6:e20594. https://doi.org/10.1371/journal.pone.0020594. [PubMed]

19. Morgan P, Van Der Graaf PH, Arrowsmith J, Feltner DE, Drummond KS, Wegner CD, Street SD. Can the flow of medicines be improved? Fundamental pharmacokinetic and pharmacological principles toward improving Phase II survival. Drug Discov Today. 2012; 17:419-424. https://doi. org/10.1016/j.drudis.2011.12.020. [PubMed]

20. Weissleder R, Pittet MJ. Imaging in the era of molecular oncology. Nature. 2008; 452:580-589. https://doi. org/10.1038/nature06917. [PubMed]

21. Roopenian DC, Akilesh S. FcRn: the neonatal Fc receptor comes of age. Nat Rev Immunol. 2007; 7:715-725. https:// doi.org/10.1038/nri2155. [PubMed]

22. Waaijer SJ, Warnders FJ, Stienen S, Friedrich M, Sternjak A, Cheung HK, van Scheltinga AG, Schröder CP, de Vries EG, Lub-de Hooge MN. Molecular Imaging of Radiolabeled Bispecific T-Cell Engager ${ }^{89} \mathrm{Zr}-\mathrm{AMG} 211$ Targeting CEAPositive Tumors. Clin Cancer Res. 2018; 24:4988-96. https:// doi.org/10.1158/1078-0432.CCR-18-0786. [PubMed]

23. Hipp S, Tai YT, Blanset D, Deegen P, Wahl J, Thomas O, Rattel B, Adam PJ, Anderson KC, Friedrich M. A novel 
BCMA/CD3 bispecific T-cell engager for the treatment of multiple myeloma induces selective lysis in vitro and in vivo. Leukemia. 2017; 31:1743-1751. https://doi. org/10.1038/leu.2016.388. [PubMed]

24. Seckinger A, Delgado JA, Moser S, Moreno L, Neuber B, Grab A, Lipp S, Merino J, Prosper F, Emde M, Delon C, Latzko M, Gianotti R, et al. Target Expression, Generation, Preclinical Activity, and Pharmacokinetics of the BCMA-T Cell Bispecific Antibody EM801 for Multiple Myeloma Treatment. Cancer Cell. 2017; 31:396-410. https://doi. org/10.1016/j.ccell.2017.02.002. [PubMed]

25. Mandikian D, Takahashi N, Lo AA, Li J, Eastham-Anderson J, Slaga D, Ho J, Hristopoulos M, Clark R, Totpal K, Lin K, Joseph SB, Dennis MS, et al. Relative Target Affinities of T-Cell-Dependent Bispecific Antibodies Determine Biodistribution in a Solid Tumor Mouse Model. Mol Cancer Ther. 2018; 17:776-785. https://doi.org/10.1158/1535-7163. MCT-17-0657. [PubMed]

26. Warnders FJ, Waaijer SJ, Pool M, Lub-de Hooge MN, Friedrich M, Terwisscha van Scheltinga AG, Deegen P, Stienen SK, Pieslor PC, Cheung HK, Kosterink JG, de Vries EG. Biodistribution and PET Imaging of Labeled Bispecific T Cell-Engaging Antibody Targeting EpCAM. J Nucl Med. 2016; 57:812-817. https://doi.org/10.2967/ jnumed.115.168153. [PubMed]

27. Waaijer SJH, Kok IC, Eisses B, Schroder CP, Jalving M, Brouwers AH, Lub-de Hooge MN, de Vries EGE. Molecular Imaging in Cancer Drug Development. J Nucl Med. 2018; 59:726-732. https://doi.org/10.2967/jnumed.116.188045. [PubMed]

28. Dahlen E, Veitonmaki N, Norlen P. Bispecific antibodies in cancer immunotherapy. Ther Adv Vaccines Immunother. 2018; 6:3-17. https://doi.org/10.1177/2515135518763280. [PubMed]
29. Liu Z, Li Z. Molecular imaging in tracking tumor-specific cytotoxic T lymphocytes (CTLs). Theranostics. 2014; 4:990-1001. https://doi.org/10.7150/thno.9268. [PubMed]

30. Larimer BM, Wehrenberg-Klee E, Dubois F, Mehta A, Kalomeris T, Flaherty K, Boland G, Mahmood U. Granzyme B PET Imaging as a Predictive Biomarker of Immunotherapy Response. Cancer Res. 2017; 77:23182327. https://doi.org/10.1158/0008-5472.CAN-16-3346. [PubMed]

31. Seo JW, Tavare R, Mahakian LM, Silvestrini MT, Tam S, Ingham ES, Salazar FB, Borowsky AD, Wu AM, Ferrara KW. CD8(+) T-Cell Density Imaging with (64)CuLabeled Cys-Diabody Informs Immunotherapy Protocols. Clin Cancer Res. 2018; 24:4976-4987. https://doi. org/10.1158/1078-0432.CCR-18-0261. [PubMed]

32. Tavare R, Escuin-Ordinas H, Mok S, McCracken MN, Zettlitz KA, Salazar FB, Witte ON, Ribas A, Wu AM. An Effective Immuno-PET Imaging Method to Monitor CD8Dependent Responses to Immunotherapy. Cancer Res. 2016; 76:73-82. https://doi.org/10.1158/0008-5472.CAN15-1707. [PubMed]

33. D'Alessandria C, di Gialleonardo V, Chianelli M, Mather SJ, de Vries EF, Scopinaro F, Dierck RA, Signore A. Synthesis and optimization of the labeling procedure of 99mTc-HYNIC-interleukin-2 for in vivo imaging of activated T lymphocytes. Mol Imaging Biol. 2010; 12:539546. https://doi.org/10.1007/s11307-009-0285-1. [PubMed] 